\title{
CHFS: Parallel Consistent Hashing File System for Node-local Persistent Memory
}

\author{
Osamu Tatebe \\ tatebe@cs.tsukuba.ac.jp \\ University of Tsukuba \\ Tsukuba, Ibaraki, Japan \\ Kohei Hiraga \\ hiraga@ccs.tsukuba.ac.jp \\ University of Tsukuba \\ Tsukuba, Ibaraki, Japan
}

\author{
Kazuki Obata \\ obata@hpcs.cs.tsukuba.ac.jp \\ University of Tsukuba \\ Tsukuba, Ibaraki, Japan \\ Hiroki Ohtsuji \\ ohtsuji.hiroki@fujitsu.com \\ Fujitsu Limited \\ Kawasaki, Kanagawa, Japan
}

\begin{abstract}
This paper proposes a design for CHFS, an ad hoc parallel file system that utilizes the persistent memory of compute nodes. The design is based entirely on a highly scalable distributed key-value store with consistent hashing. CHFS improves the scalability of parallel data-access performance and metadata performance in terms of the number of compute nodes by eliminating dedicated metadata servers, sequential execution, and centralized data management. The implementation efficiently utilizes multicore and manycore CPUs, high-performance networks, and remote direct memory access by the Mochi-Margo library. With a 4-node persistent memory cluster, CHFS performs 9.9 times better than the state-of-the-art DAOS distributed object storage and 6.0 times better than GekkoFS on the IOR hard write benchmark. Regarding scalability, CHFS displays better scalability and performance for both bandwidth and metadata compared with BeeOND and GekkoFS. CHFS is a promising building block for HPC storage layers.
\end{abstract}

\section{CCS CONCEPTS}

- Information systems $\rightarrow$ Distributed storage.

\section{KEYWORDS}

Parallel file system, Distributed key-value store, Persistent memory

\section{ACM Reference Format:}

Osamu Tatebe, Kazuki Obata, Kohei Hiraga, and Hiroki Ohtsuji. 2022. CHFS: Parallel Consistent Hashing File System for Node-local Persistent Memory. In International Conference on High Performance Computing in Asia-Pacific Region (HPC Asia2022), fanuary 12-14, 2022, Virtual Event, Japan. ACM, New York, NY, USA, 10 pages. https://doi.org/10.1145/3492805.3492807

\section{INTRODUCTION}

Leadership supercomputers are utilized not only for compute-intensive computational science applications but also for data-intensive big

Permission to make digital or hard copies of all or part of this work for personal or classroom use is granted without fee provided that copies are not made or distributed for profit or commercial advantage and that copies bear this notice and the full citation on the first page. Copyrights for components of this work owned by others than ACM must be honored. Abstracting with credit is permitted. To copy otherwise, or republish, to post on servers or to redistribute to lists, requires prior specific permission and/or a fee. Request permissions from permissions@acm.org.

HPC Asia2022, January 12-14, 2022, Virtual Event, Japan

(C) 2022 Association for Computing Machinery.

ACM ISBN 978-1-4503-8498-8/22/01 . .\$15.00

https://doi.org/10.1145/3492805.3492807 data analysis and machine learning applications with large-scale data. Storage performance is always a problem because the CPU/GPU performance improves faster than storage performance. To reduce this performance gap, leadership supercomputers including Summit [19] at Oak Ridge National Laboratory, ABCI at AIST, Tsubame at Tokyo Tech., and Cygnus at the University of Tsukuba introduced local storage into compute nodes, such as NVMe SSD and persistent memory. However, efficient utilization of node-local storage is a challenging issue that has been addressed by several groups. An ad hoc distributed file system is a temporal file system that is available during job allocation, which uses the local storage of compute nodes. Existing designs of ad hoc distributed file systems rely either on block-based storage utilizing a local file system or a database with a log-structured merge-tree (LSM tree) data structure. Applying these approaches to byte-addressable persistent memory is not optimal for efficiently utilizing the performance benefit of persistent memory [9].

This paper proposes a design for CHFS, an ad hoc parallel file system that uses node-local persistent memory. The major features of the design include exploiting the low latency and high bandwidth characteristics of byte-addressable persistent memory, as well as the high scalability of parallel file access and metadata performance in terms of the number of compute nodes. The design utilizes a persistent in-memory key-value store to exploit low latency and high bandwidth. Because persistent memory is byte-addressable, it is not necessary to use a data structure optimized for a block device. Moreover, dedicated metadata servers are not used to reduce metadata access overhead. Historically, storage performance has been improved by decoupling metadata management and file data management $[3,8,32]$. In this case, the client processes can access the file data in parallel after accessing the metadata. In contrast, the single metadata server approach has scalability limitations in terms of the number of client processes. To overcome this limitation, distributed metadata servers have been investigated [10, 20, 24]. To further reduce the metadata access overhead, this study proposes accessing the file data directly without accessing the metadata. Moreover, to avoid limiting scalability, the study excluded sequential execution and centralized data management. The proposed file system is based on a highly scalable distributed key-value store that uses consistent hashing. CHFS is carefully designed so as not to kill the performance of the underlying distributed key-value store.

The contribution of this research includes 
- design of an ad hoc parallel file system with scalable metadata performance and scalable file access performance for persistent memory of compute nodes in HPC,

- a simple file system design based on a distributed key-value store that can be flexibly applied to other distributed keyvalue stores,

- open-source implementation to efficiently utilize multicore and manycore CPUs, high-performance networks, and remote direct memory access (RDMA) that can be used for research, experimentation, and production,

- a flexible backend design that uses not only a persistent inmemory key-value store but also a POSIX file system for flash and other block devices.

\section{RELATED WORK}

The performance and characteristics of persistent memory have been reported by Yang [39]. A programming development kit for persistent memory (PMDK) has been developed to provide a portable programming interface [21,27]. Pmemkv [22] is a library for a persistent in-memory key-value store in the PMDK.

DAOS is a state-of-the-art distributed object storage system designed for persistent memory and flash devices $[4,15]$. The DAOS of the Intel Wolf system achieved the best IO performance on the IO500 list of June 2020 [11]. It is not expected to be used as a temporally distributed file system that exploits node-local persistent memory considered in this paper. It assumes dedicated storage nodes with persistent memory and flash devices.

Orion [38] is a distributed file system for persistent memory. It exploits RDMA to improve performance and has a single metadata server, which limits the scalability of the metadata performance when the number of client processes increases.

Octopus [16] is a distributed file system for persistent memory with distributed metadata management. It proposes a collectdispatch transaction to support file system operations such as mkdir, mknod, and rmdir in distributed transactions. Meanwhile, CHFS does not require distributed transactions because each file system operation accesses only one key-value pair, which is more efficient than the Octopus with respect to the performance.

There are several ad hoc distributed file systems [5] such as Gfarm/BB [33], BurstFS [37], UnifyFS [18], BeeOND [34] and GekkoFS [36]. Gfarm/BB exploits storage locality. This creates a file in node-local storage. To manage the file locations, it has a single metadata server with hot spares. BurstFS and UnifyFS also exploit the locality of storage to write data in node-local storage in a logstructured format. The metadata are managed by a distributed key-value store. These systems mostly focus on checkpointing write-intensive workloads, while CHFS is optimized for both read and write operations. BeeOND uses distributed metadata servers to improve metadata performance, while CHFS does not use dedicated metadata servers to further improve metadata performance. GekkoFS does not have dedicated metadata servers. It only has storage servers where the metadata are stored using RocksDB [6], and the file data are stored in a local file system. RocksDB is not optimized for persistent memory [17]. In GekkoFS, the metadata and file data are distributed by the hash value modulo. GekkoFS is similar to CHFS; however, CHFS is entirely designed on a distributed key-value store, where all the data are stored. CHFS has a simple design that can be flexibly applied to other distributed key-value stores. With consistent hashing, which minimizes data movement when a server of a distributed key-value store joins or leaves, most data are still available with CHFS even after a server joins or leaves.

A burst buffer was introduced to absorb bursty parallel write access to a parallel file system in HPC applications [2]. The burst buffer is mostly used as a write-back cache. In production systems, a burst buffer is deployed via dedicated storage nodes. Infinite Memory Engine (IME) [7] can be shared among multiple users and processes and used as a temporal storage area for in-transit data analysis [26]. IME relies on a backend parallel file system for metadata management, which limits metadata performance.

SymphonyFS [19] was proposed as a write-back cache for a parallel file system. It utilizes the node-local NVMe SSD for temporal cache storage and supports a single-shared-file access pattern using block-based log-structured cache management. It also relies on the backend parallel file system for metadata management, which limits metadata performance. The metadata performance of a BeeGFSbased caching file system [1] is similarly limited.

Persistent client caching in Lustre [23] is another way to utilize node-local storage. It applies a hierarchical storage management mechanism to cache files in node-local storage. Because it caches complete files, the single-shared-file access performance does not improve.

There are several proposals for distributed hash tables using peer-to-peer technology. Chord [31] is a scalable look-up protocol for consistent hashing. When it is distributed among $N$ nodes, the original consistent hashing [12] needs to know the list of all $N$ nodes in all nodes. Chord requires $\log (N)$ entries for a routing table in each node and $\log (N)$ steps to look up a target node. Chord and other peer-to-peer systems are optimized to improve the scalability of the number of peers, while CHFS is optimized to improve the performance of metadata and parallel data-access performance.

\section{DESIGN OF CHFS}

The design goals of CHFS, an ad hoc temporal parallel file system for node-local persistent memory in HPC, are as follows:

- to reduce metadata access overhead and improve the scalability of metadata performance in terms of the number of compute nodes

- to improve the scalability of parallel data-access performance in terms of the number of compute nodes.

To meet these requirements, CHFS uses the performance of persistent memory and avoids centralized data management.

\subsection{Persistent memory}

Persistent memory can be directly accessed when configured in the fsdax (Filesystem DAX) mode or devdax (Device DAX) mode. The fsdax mode creates a block device. Direct access to persistent memory in fsdax mode is possible using an mmap system call with the dax mount option. The devdax mode creates a character device. Persistent memory in the devdax mode can be accessed directly. Although the devdax mode cannot use the file system interface, it 
performs better than the fsdax mode because it does not incur the overhead of the file system.

In case of failure, the status of persistent memory may be inconsistent, causing failure to recover. To avoid this issue, the persistent memory is updated using transactions. Persistent data structures are designed using transactions. A promising data structure for storage is a persistent in-memory key-value store. To efficiently utilize the low latency and high bandwidth of persistent memory, CHFS accesses persistent memory through the key-value interface.

\subsection{Design of file system}

A distributed key-value store is obtained by partitioning a key-value store horizontally. Such a store has excellent scalability properties in terms of access performance with respect to the number of servers. CHFS is designed to store file system metadata and file data in a distributed key-value store. File system metadata comprise a hierarchical namespace, such as directory trees and entry information such as mode, time, and owner.

There are two ways to manage a hierarchical namespace in a distributed key-value store. A hierarchical namespace can be managed as a single flat namespace in distributed object stores, such as S3 [29], using a full path as the key. The '/' character in the key serves as the separator of the directory path. This method has the advantage of accessing a target file without traversing the hierarchical directories. However, this design is problematic when moving or renaming a directory. In this case, the keys of all entries under the directory must be changed. Another method is to manage the hierarchical namespace in a distributed key-value store via a unique directory ID. Each entry is referenced by the parent directory ID and entry name [10, 14, 24]. For example, let the '/usr' directory ID be 3 . The '/usr/local' directory is referenced by '3/local' when assuming a directory ID and the entry name is separated by ' $/$ '. In this case, an entry is examined by traversing the hierarchical directories. To look up the '/usr/local' directory, first, the '/usr' directory is looked up and directory ID 3 is obtained. Then, '3/local' is looked up. The merit of moving or renaming a directory using this method is that the operational cost is $O(1)$. This look-up cost can be optimized by caching the look-up results.

To reduce the metadata access overhead, CHFS uses the former method, which takes the full path as the key. In this case, the file data can be accessed by a single put/get operation to an underlying distributed key-value store.

In a file system, a file is opened before it is accessed. However, a distributed key-value store does not have an open operation. According to the POSIX semantics, a file can be accessed while open, even after the file is removed. Support for these semantics in a key-value store is not straightforward and may cause a high overhead. CHFS does not strictly support these semantics because HPC applications do not require such a rare case. Instead, it manages the open status in the client library and assigns a file descriptor at the open operation. For read and write operations, the specified file descriptor is converted to a full path to access the data.

In HPC, a single file can be accessed in parallel by multiple parallel processes. To improve the parallel file access performance of a single file, a file is divided into fixed-size chunks. Because the optimal chunk size depends on the access pattern of the file, the

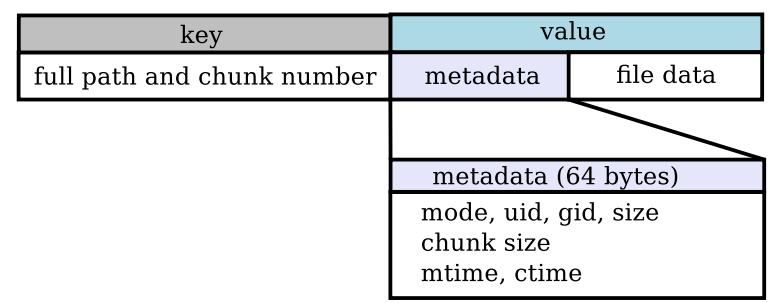

Figure 1: Design format of a key and a value in CHFS. A key is a full path name and a chunk number. A value is metadata and file data. There is only one format in CHFS.

size is specified when a file is created. CHFS assumes the use of persistent memory and the chunk size is specified in bytes. Because a file is divided into chunks, a key comprises a full path and chunk number.

The file system also needs to manage entry information apart from a hierarchical namespace, such as mode, user id (uid), group id (gid), file size, modification timestamp (mtime), and status change timestamp (ctime). In CHFS, a chunk size is managed as metadata because a file is divided into chunks and each file has a different chunk size. These metadata are stored as a value in a distributed key-value store. Figure 1 shows the design of the format of the key and the corresponding value in the CHFS. A key is a full path name and chunk number and a value is the entry information (metadata) and file data. This key-value pair corresponds to a file chunk or directory. There is only one format, and all data in the CHFS are in this format. A directory does not use the chunk number, chunk size, size, or file data in the format. A regular file may consist of several chunks. The entry information is basically for each chunk and not for the whole file. The file size in this format is the file size of the file chunk. Note that CHFS does not manage the total file size or total chunk number because the metadata update of these fields is a serialized execution that requires locking, or an atomic operation in parallel file accesses, thus limiting scalability. Instead, the CHFS counts the number of chunks at the stat operation to determine the total file size. This design choice improves the parallel write and read performance, rather than the file stat performance.

\subsection{Server interface}

Server interfaces are prepared to consistently access and update the metadata via file system operations. As described in Section 3.2, the CHFS stores a part of the metadata in the value part. This metadata part should be managed by a server, not by a client library, because metadata are critical for file system management.

\subsection{Consistent hashing}

CHFS distributes key-value pairs in a distributed key-value store by using consistent hashing. The consistent hashing feature moves the minimum size of the data when a server joins or leaves. CHFS uses ring-based consistent hashing. Each server has a list of all other servers because the number of servers is at most in the range of several tens of thousands. In this way, each server can look up a target server locally without any communication, which is more efficient than the Chord approach. 
The CHFS uses a hash function to distribute key-value pairs. The servers are distributed according to the hash value in a ring. Each server takes charge of the range from the hash value of the previous server in ring +1 to the hash value of the self server. When the number of servers is small, the range size of each server tends to be unbalanced. To balance the range size, i.e., the server load, virtual servers are introduced [12]. In this case, each server serves several virtual servers, essentially increasing the number of servers required to balance the range size. Moreover, this study proposes the use of a virtual name for each server to control the server hash value for better load balancing.

\subsection{Local storage interface}

We currently use the pmemkv library for persistent in-memory keyvalue stores in the PMDK because it is publicly available. Pmemkv supports both fsdax and devdax in persistent memory mode. The directory listing (readdir) operation is supported by a range search using a parent directory path.

For storage systems that do not support a key-value interface, the POSIX backend interface is used for the design of the keyvalue interface, such that a key is a file name and a value refers to file data. A key in CHFS is a full path and chunk number, which includes the '/' character; thus a put operation requires the creation of intermediate directories including a full path.

\section{IMPLEMENTATION OF CHFS}

The CHFS consists of a CHFS client library and a CHFSD server. The client library provides a file system interface. The CHFSD is the server of a distributed key-value store.

CHFS was implemented in the Mochi-Margo library [25]. MochiMargo is a library of network services based on the Mercury communication library [30] for remote procedure call (RPC) and RDMA, and Argobots lightweight thread library [28]. Mochi-Margo provides a blocking RPC programming interface while implicitly using a progress loop for communication and callbacks to nonblocking communications. Mochi-Margo efficiently supports an event-driven execution model in Mercury and lightweight multithread execution. Mercury, in turn, supports several network layers including tcp, verbs, psm2 (Omni-Path), and gni (Gemini/Aries) provided by libfabric.

The RDMA feature is provided by Mercury in any network layer. CHFS uses RDMA when the data size is larger than a specified threshold because the data transfer of small message is not efficient in RDMA.

\subsection{Distributed key-value store}

A distributed key-value store comprises a pool of CHFSD servers. The CHFSD servers are connected in a ring structure to broadcast messages. A new CHFSD server can join an existing pool by calling a join RPC to any CHFSD server in the pool. When a new CHFSD server joins an existing pool, an election RPC is sent to create a new list of servers and determine a new coordinator [35]. The election RPC sends the address of the self server to the next server in the ring. When a CHFSD server receives the election RPC from the previous server in the ring, it sends a list comprising the received list of servers and the address of the self server. As the election
RPC goes around the ring, the server list consists of all servers. The server list is transferred by the coordinator RPC for another round to provide all servers with the server list. The maximum value in a string comparison determines the coordinator server. The coordinator periodically sends a heartbeat RPC around the ring. When a server failure is encountered, an election RPC is sent to create a new list of servers and determine a new coordinator.

Consistent hashing is used to determine the range of the CHFSD server keys that are in charge; a hash function is used for this purpose. Each CHFSD server is notified of the list of all servers by the coordinator RPC. Each server then calculates a hash value, the order of which is used to sort the servers. A target server is looked up using the hash value of a key consisting of a full path and a chunk number, which can be binary or linearly dependent on the number of total servers.

Figure 2 shows the major client interfaces for the RPC to a CHFSD server. $f s_{-}$create creates a file or directory by specifying a key and file metadata; mode and chunk size. When the requested server is in charge of the key, the file or directory is created locally; otherwise, the server forwards an fs_create RPC to a server in charge. This forwarding mechanism was applied to all RPCs.

$f_{s}\{$ write,read $\}$ writes or reads file data in a file chunk by specifying a key. With the usual file system interface, the open operation provides a file descriptor, which is used to read or write data. Because the backend of the CHFS is a distributed key-value store, the open status is managed by a client library. The client library converts a file descriptor into a key. When the buffer size is large, the data are transferred over the RDMA interface, which requires an additional client address for RDMA operations. In the case of a write, a new chunk may be created, which is why fs_write requires an additional argument for mode and chunk size.

$f$ s_readdir sends a path to a server and obtains entry names and struct stat data structures in the specified directory path, which are passed to a filler function in the client library. Because entries are distributed among all CHFSD servers, this RPC should be sent to all servers.

fs_remove removes a file chunk or directory by specifying a key. In file removal, all chunks should be removed. When the file size is large, it is inefficient to remove all chunks successively. To optimize such a case, we introduce an asynchronous unlink_chunk_all RPC. This asynchronous RPC requests the removal of all chunks of a specified file stored on a CHFSD server. After the asynchronous RPC is sent to all servers, an unlink_chunk_all_wait RPC is used to wait for completion.

\subsection{Pmemkv}

Pmemkv is a library for a persistent in-memory key-value store. Figure 3 shows the major APIs of pmemkv. Pmemkv ensures crash consistency using transactions in the pmemobj library in the case of a sudden crash or power outage.

pmemkv_open() opens a database for a persistent in-memory keyvalue store. At the time of writing this paper (July 2021), the cmap concurrent hashmap was available for the persistent key-value engine. The put/get/remove operations were executed in parallel. In contrast, the cmap engine does not support the range query because the key-value pairs are not sorted. 


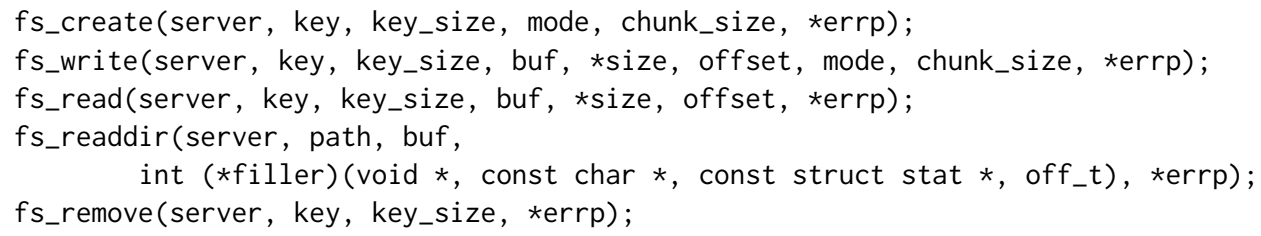

Figure 2: Major client interfaces for RPC to CHFSD server. Most interfaces require the key and key size to specify a file chunk or directory. File system metadata such as mode and chunk size are specified as arguments for a new file chunk or directory.

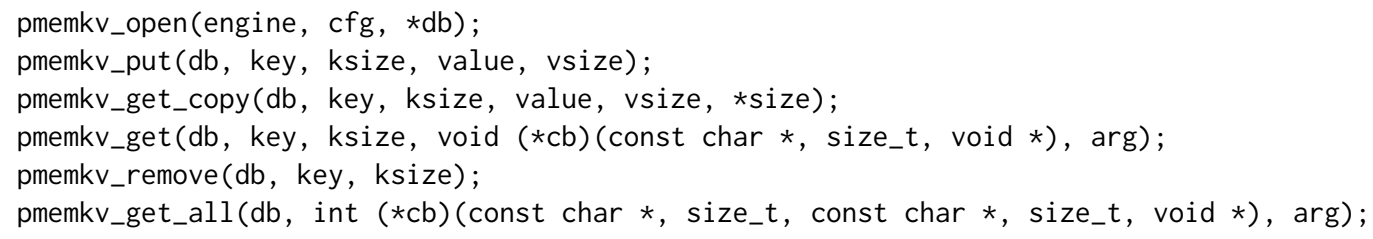

Figure 3: Major interfaces of pmemkv. Put inserts a key-value pair. To avoid data copy, we need to use get not get_copy. Get_all calls a callback function cb for all key-value pairs.

pmemkv_put() inserts a key-value pair. pmemkv_get_copy() copies a value to a specified buffer. pmemkv_get() calls a specified callback function with the address of the value and the value size. To avoid memory copy, pmemkv_get() is used in the CHFS.

A readdir RPC must return entries in a specified directory. However, the cmap engine does not support range queries. Currently, CHFS uses pmemkv_get_all() to execute a callback function for all entries. Another possible solution is to create a key-value database for each directory. However, this solution is not realistic when the space utilization of persistent memory is considered. The minimum database size of pmemkv is $8 \mathrm{MiB}$, which limits the maximum number of directories. In addition, there is no way to determine the appropriate database size for each created directory.

\subsection{POSIX backend}

The POSIX backend key-value interface is designed and implemented for storage not used by pmemkv. In general, a key is a file name, and the value refers to the file data. Put(key, value) is implemented using the following pseudocode:

$f d=\operatorname{open}($ key $)$;

write (fd, value);

close $(f d)$;

POSIX I/O system calls such as open, write, and close may block an execution thread. To avoid this, the ABT-IO [13] was utilized. ABT-IO provides Argobots-aware wrappers to POSIX I/O system calls. It delegates blocking I/O system calls to a dedicated set of threads and executes another runnable thread.

In CHFS, the value of a key-value pair includes metadata, such as mode, uid, gid, file size, mtime, ctime, and chunk size. However, except for chunk size, a file in the POSIX file system has the same metadata. Thus, it is not necessary to store the metadata in a value. There are two possibilities for chunk size. One is to store an extended attribute, and the other is to store in a value. We implement both options such that either one can be selected at the time of configuration. In CHFS, the key is a full path and a chunk number.
Because a full path includes a '/' character, a put operation must create intermediate directories as required and a remove operation should be performed as appropriate.

\subsection{Client interface}

CHFS has a POSIX-like libchfs client library and a chfuse FUSEbased mount program. The library supports a subset of the POSIX interface required by HPC applications, including open, close, read, write, pread, pwrite, fsync, unlink, mkdir, rmdir, stat, and readdir. Chfuse, supported by libchfs, provides equivalent functionality.

A list of CHFSD servers can be specified as the CHFS_SERVER environment variable. The list does not need to be complete. A subset of CHFSD servers, or even a single server in a server pool, should be sufficient. At the time of initialization, the client library accesses a randomly chosen CHFSD server to obtain the current server list. By selecting a contact server randomly, communication issues related to parallel applications are avoided during initialization.

The client library utilizes the server list to resolve the CHFSD server in charge of a specified key-value pair, thus avoiding communication concentration and unnecessary communication forwarding. Even when this resolution fails, the RPC request is forwarded to an appropriate server. This server list is also used for readdir and unlink operations, which in turn send readdir and unlink_chunk_all RPCs to all servers. The client library periodically updates the server list to address configuration changes.

\section{PERFORMANCE EVALUATION}

This section evaluates the performance of persistent memory and CHFS as follows:

(1) Performance evaluation of the persistent memory,

(2) Performance comparison with DAOS and GekkoFS,

(3) Performance comparison with Gfarm/BB and BeeOND,

(4) Scalability evaluation of CHFS. 


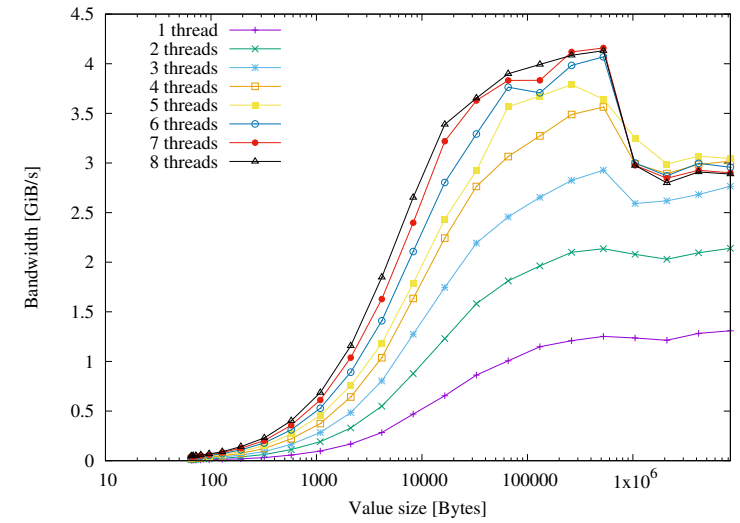

Figure 4: The put bandwidth of pmemkv in fsdax. The best bandwidth is $4.2 \mathrm{GiB} / \mathrm{s}$ with 7 threads when the value size is $512 \mathrm{KiB}$.

In this experiment, two evaluation platforms are used. One platform is a 4-node Chris cluster connected by InfiniBand HDR100, where each node has a $2.3 \mathrm{GHz} 16$-core Xeon 5218, 96GiB DDR43200 memory, and $768 \mathrm{GiB}$ Optane DC persistent memory. The other platform is a 78-node Cygnus supercomputer at the University of Tsukuba, where each node has two sockets of $2.6 \mathrm{GHz} 12$-core Xeon 6126, 192GiB DDR4-2666 memory, and 3.2TB Intel SSD DC P4610. The compute node of the Cygnus supercomputer does not have persistent memory. For both systems, CentOS 7 and Open MPI were used.

\subsection{Performance evaluation of persistent memory}

Persistent memory performance was evaluated on one node of the Chris cluster using the pmemkv library in the fsdax and devdax modes and the POSIX interface. The figures summarize the results.

Figure 4 displays the put bandwidth of pmemkv in the fsdax mode. In this experiment, the key size was 20 bytes. The bandwidth was measured by changing the value size and number of threads. The put operation was executed for 40 seconds to avoid exceeding the capacity of the persistent memory. In the case of one thread, the maximum bandwidth was approximately $1.3 \mathrm{GiB} / \mathrm{s}$. As the number of threads increased, the bandwidth improved. With seven threads, the maximum bandwidth was $4.2 \mathrm{GiB} / \mathrm{s}$ for a value size of $512 \mathrm{KiB}$. The performance degraded when the value size was increased to 1 MiB.

Figure 5 displays the put bandwidth of pmemkv in the devdax mode. The figure displays the same trend as in the case of fsdax but the physical bandwidth was much higher. In the case of one thread, the maximum bandwidth was approximately $2.0 \mathrm{GiB} / \mathrm{s}$. With eight threads, the maximum bandwidth was $8.5 \mathrm{GiB} / \mathrm{s}$ for a value size of $512 \mathrm{KiB}$. The performance degraded when the value size was increased to $1 \mathrm{MiB}$, similar to the case of fsdax.

Figure 6 displays the write bandwidth of the POSIX interface using the IOR benchmark on XFS with the dax mount option in the fsdax mode. In this experiment, the write bandwidth was measured using the file-per-process access pattern by changing the block size

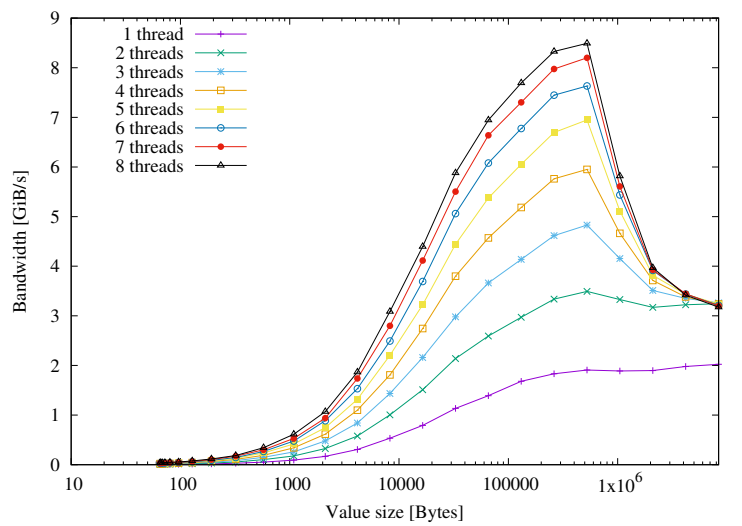

Figure 5: The put bandwidth of pmemkv in devdax. The best bandwidth is $8.5 \mathrm{GiB} / \mathrm{s}$ with 8 threads when the value size is $512 \mathrm{KiB}$.

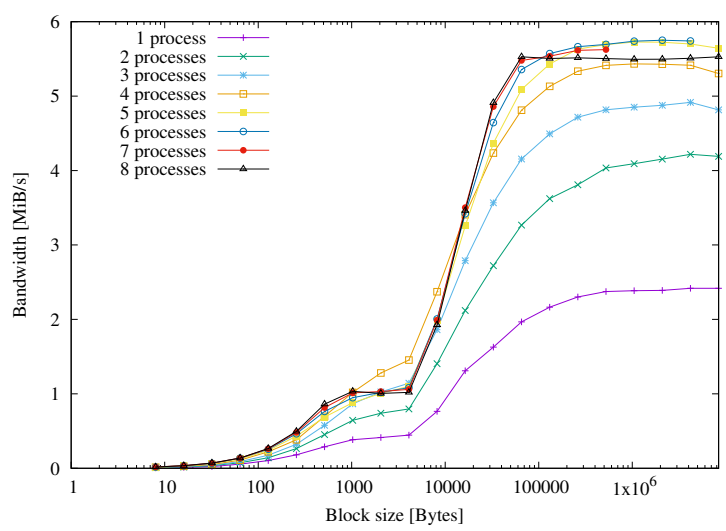

Figure 6: The write bandwidth of POSIX interface using IOR on XFS in fsdax. The best bandwidth is $5.8 \mathrm{GiB} / \mathrm{s}$ with 6 processes when the block size is $2 \mathrm{MiB}$, which is less than the case of pmemkv in devdax.

and the number of processes. The write operation was executed for 40 seconds. In the case of one process, the maximum bandwidth was $2.4 \mathrm{GiB} / \mathrm{s}$. As the number of processes increased, the performance improved. With six processes, the maximum bandwidth was 5.8 $\mathrm{GiB} / \mathrm{s}$ for a block size of $2 \mathrm{MiB}$, which is less than the case of pmemkv in the devdax mode. In this case, the bandwidth was not degraded when the block size was increased to $1 \mathrm{MiB}$.

In the following experiments, the devdax mode was used because it performed the best.

\subsection{Comparison with DAOS and GekkoFS}

CHFS, DAOS, and GekkoFS were evaluated on the 4-node Chris cluster using the IO500 benchmark. Although DAOS is not an ad hoc temporal file system, the performance comparison helped clarify the persistent memory utilization of CHFS because the design and 


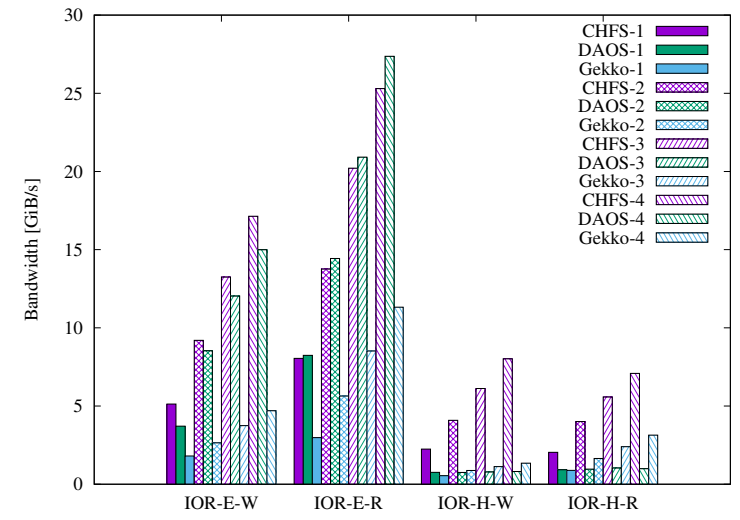

Figure 7: IO500 bandwidth performance of CHFS, DAOS and GekkoFS. IOR-E-W and IOR-E-R denote IOR easy write and read, respectively. IOR-H-W and IOR-H-R denote IOR hard write and read, respectively. CHFS displays the best performance except for the IOR easy read case.

implementation of DAOS are carried out by the production vendor of persistent memory.

The IO500 benchmark consists of the bandwidth measurement of IOR and the metadata performance measurement of the MDtest and find [11]. The bandwidth is measured in two access patterns: easy and hard. The easy case is a file-per-process access pattern. Each process accesses its own file. The hard case is a single-shared-file access pattern with a block size of 47,008 bytes. The hard access pattern is more difficult than the access patterns of real applications.

Regarding metadata performance, there are two access patterns. The easy case is a unique directory pattern in which each process creates, stats, and deletes the empty files in its own directory. The hard case is a shared directory pattern such that all processes create, stat, read, and delete files of 3,901 bytes in a single shared directory. The find benchmark finds files that are newer than a temporal file, have a size of 3,901 bytes, and have a name pattern of '* $01^{*}$.

Figure 7 shows the bandwidth performance of CHFS, DAOS, and GekkoFS. The DAOS version is 1.0.1 and the DFS driver is used for IOR. In addition, the PMEMOBJ_CONF environment variable was set to 'prefault.at_open $=1$; prefault.at_create $=1$ ' to minimize the impact of page faults. Neither data replication, nor erasure coding was used for the best DAOS performance. GekkoFS is the latest version committed on March 30, 2021. GekkoFS uses the sockets network layer over InfiniBand to avoid runtime errors. CHFS- $n$, DAOS- $n$, and Gekko- $n$ use $n$ nodes for client and server process execution, respectively. DAOS does not regularly assume that the client and server processes are executed on the same node; however, CHFS does. Therefore, this performance evaluation was carried out under the same node assumption. In all cases, the number of processes per node was eight. Regarding the IO500 regulation, the write benchmark should be executed for more than 300 seconds. However, it was executed for 30 seconds not to exceed the capacity of persistent memory. For the IOR hard case, the chunk size was specified as 47,008 bytes for the CHFS and DAOS.

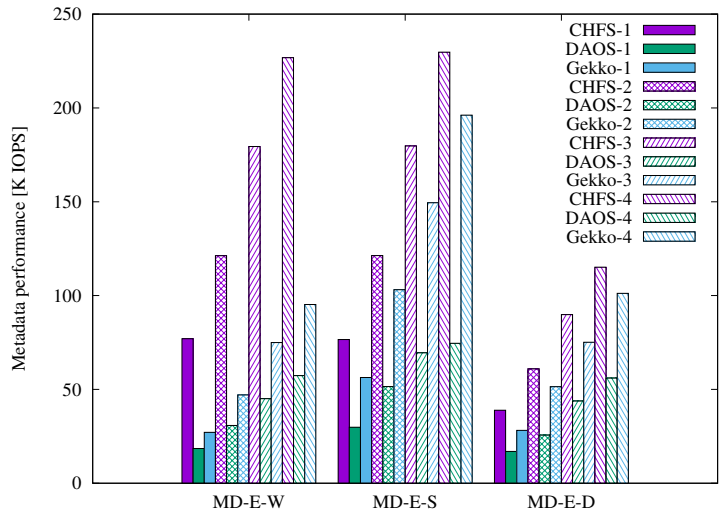

Figure 8: IO500 metadata performance of CHFS, DAOS, and GekkoFS in the easy case. MD-E-W, MD-E-S and MD-E-D denote MDtest easy write, stat, and delete, respectively. CHFS displays the best and scalable performance.

In the case of IOR easy write (IOR-E-W), CHFS shows 1.08 to 1.38 times better performance than DAOS, while in the case of IOR easy read (IOR-E-R), DAOS shows 1.02 to 1.08 times better performance than that of CHFS. DAOS has its own persistent in-memory keyvalue store, which performs better than the pmemkv utilized by CHFS. Compared to GekkoFS, CHFS displays 2.2 to 3.6 times better performance in write and read.

In the IOR hard case, CHFS shows 3.0 to 9.9 times better performance than DAOS in write (IOR-H-W), and 2.2 to 7.1 times better performance in read (IOR-H-R). CHFS shows 4.1 to 6.0 times better performance than GekkoFS in write, and 2.3 to 2.4 times better performance in read. IOR hard requires a much larger number of blocks for access because the block size is small. CHFS has a great advantage over DAOS and GekkoFS while accessing small block sizes because the CHFS access overhead is low.

Figure 8 shows the metadata performance of CHFS, DAOS, and GekkoFS in the easy case. In the MDtest easy write (MD-E-W) case, CHFS shows 3.9 to 4.2 times better performance than DAOS, and 2.4 to 2.8 times better performance than GekkoFS. In the MDtest easy stat (MD-E-S) and delete (MD-E-D) cases, CHFS shows 2.0 to 3.1 times better performance than DAOS, and 1.1 to 1.4 times better performance than GekkoFS.

Figure 9 shows the metadata performance of CHFS, DAOS, and GekkoFS in the hard case. In the MDtest hard write (MD-H-W) case, CHFS shows 3.7 to 6.0 times better performance than DAOS and GekkoFS. In the MDtest hard stat (MD-H-S) and delete (MD-H-D) cases, CHFS shows 2.4 to 5.6 times better performance than DAOS, and 1.2 to 2.4 times better performance than GekkoFS. In the MDtest hard read (MD-H-R) case, CHFS is competitive with DAOS, and displays 1.5 to 1.7 times better performance than GekkoFS. CHFS shows the best performance among all three.

\subsection{Comparison with Gfarm/BB and BeeOND}

This section compares the performance of CHFS, Gfarm/BB, and BeeOND using the Cygnus supercomputer. Because Cygnus does not have persistent memory, the evaluation was performed using 


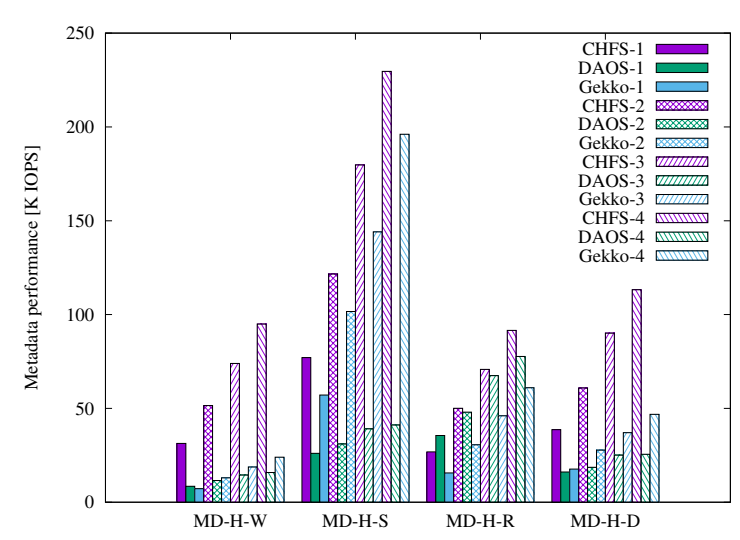

Figure 9: IO500 metadata performance of CHFS, DAOS, and GekkoFS in the hard case. MD-H-W, MD-H-S, MD-H-R and MD-H-D denote MDtest hard write, stat, read, and delete, respectively. CHFS displays the best and scalable performance.

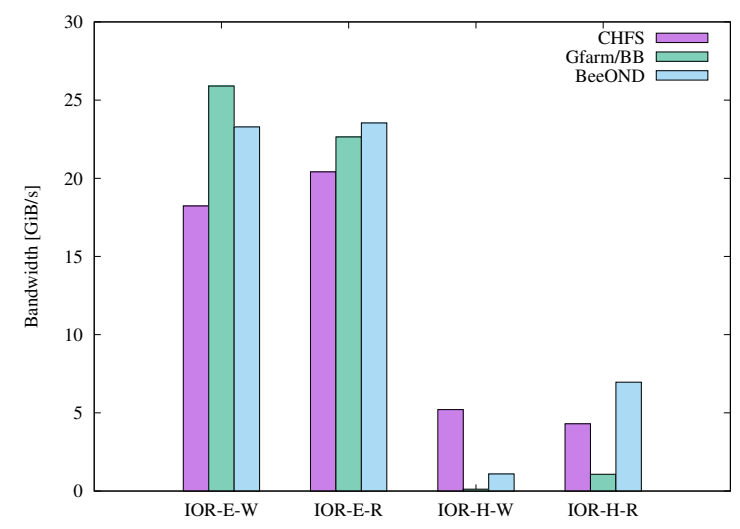

Figure 10: IO500 bandwidth of CHFS, Gfarm/BB, and BeeOND using 10 nodes / 200 processes of Cygnus supercomputer. For IOR hard case, CHFS displays consistently good performance.

the node-local NVMe SSD. Note, however, that CHFS is not optimized for SSD.

In this experiment, we used the same 10 nodes for servers and clients. The number of processes per node for the client was 20 . In this section, the write benchmarks are executed for more than 300 seconds, which conforms to the IO500 rules.

Figure 10 shows the bandwidth of the IO500 benchmark using 10 nodes. In the IOR easy case, all file systems displayed good performance. Gfarm/BB performed the best because it utilized the locality of storage access.

In the IOR hard case, CHFS displayed a consistent performance of approximately $5 \mathrm{GiB} / \mathrm{s}$. Gfarm/BB performed the worst because it did not partition the file and accessed only one server node. BeeOND performed better in the read case owing to the utilization of the server cache.

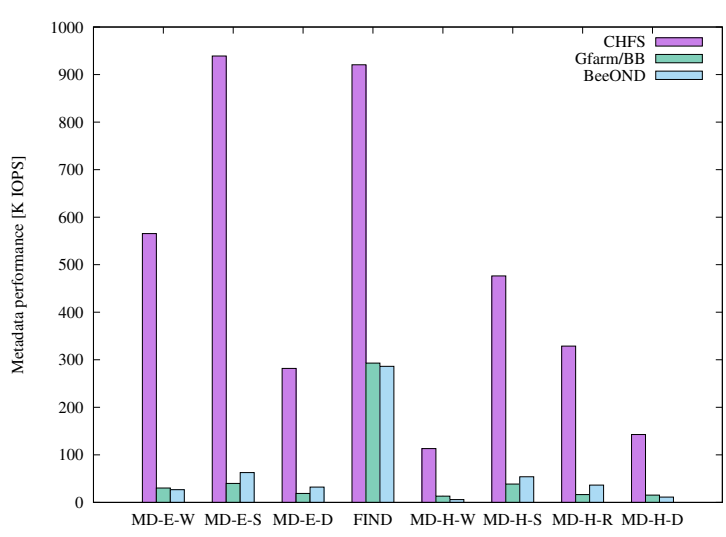

Figure 11: IO500 metadata performance of CHFS, Gfarm/BB, and BeeOND using 10 nodes / 200 processes of Cygnus supercomputer. CHFS outperformed Gfarm/BB and BeeOND.

Figure 11 displays the metadata performance of the IO500 benchmark using 10 nodes. Except for the find, CHFS shows 8.6 to 23.5 times better performance than Gfarm/BB and BeeOND. For the find, CHFS shows 3.1 to 3.2 times better performance than Gfarm/BB and BeeOND. These results display the low latency and high throughput of the metadata access of CHFS compared with those of Gfarm/BB and BeeOND.

\subsection{Scalability}

This section discusses the scalability of bandwidth and metadata performance in terms of the number of compute nodes. Figure 12 displays the IO500 bandwidth scores of CHFS, BeeOND, and GekkoFS with the number of compute nodes changing from 1 to 64 with 20 processes per node. The IO500 bandwidth score is calculated by the geometric mean of the bandwidth of the IOR easy write, IOR easy read, IOR hard write, and IOR hard read. CHFS exhibits better scalability than BeeOND. When 64 compute nodes are used, the CHFS score is 17.3 times better than that of the one compute node case, while the BeeOND score is 5.4 times better than that of the one compute node case. When using 64 nodes, GekkoFS displays device or resource busy errors when creating files in IOR easy write. Therefore, the performance of 64 nodes was not included. GekkoFS displays good scalability but is poorer than that of CHFS.

Figure 13 shows the IO500 metadata scores of the CHFS, BeeOND, and GekkoFS. The IO500 metadata score is calculated by the geometric mean of the performance of the MDtest easy write/stat/delete and MDtest hard write/stat/ read/delete and find.

CHFS exhibits better scalability than BeeOND. When using 64 compute nodes, CHFS scores 15.8 times better compared to the one compute node case, while BeeOND scores 1.5 times better compared to the one compute node case. Because CHFS does not include any sequential execution or centralized data management, the performance improves as the number of compute nodes increases. GekkoFS displays good scalability, but its performance is poorer than that of CHFS. 


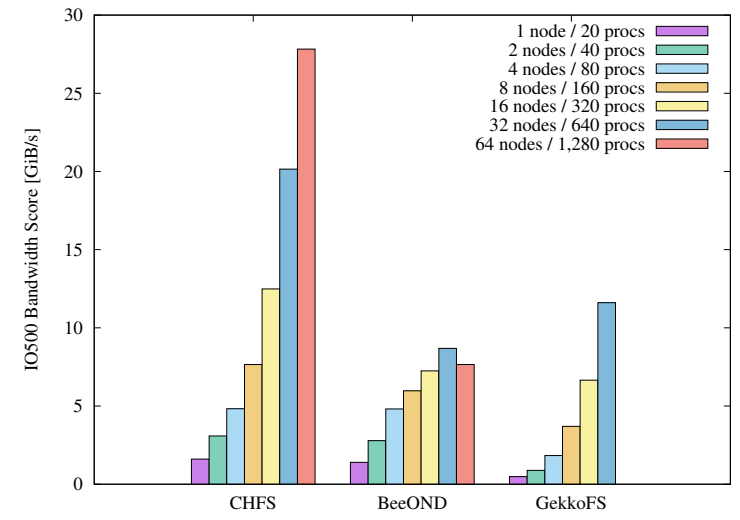

Figure 12: IO500 bandwidth score of CHFS, BeeOND, and GekkoFS on Cygnus supercomputer. CHFS exhibits much better scalability and performance.

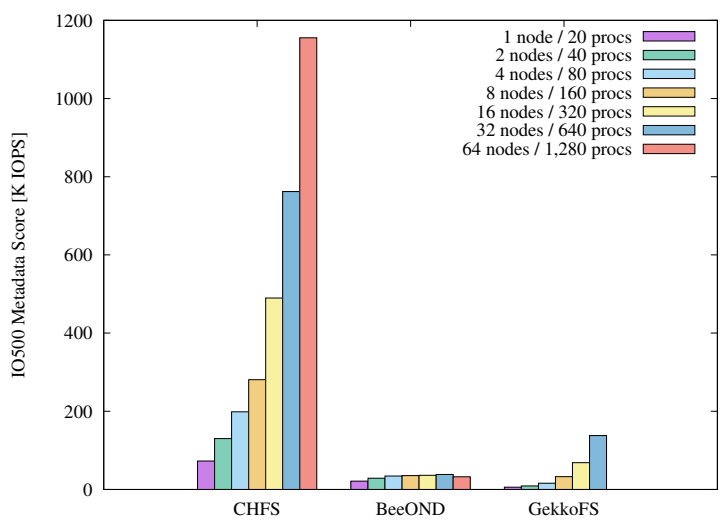

Figure 13: IO500 metadata score of CHFS, BeeOND, and GekkoFS on Cygnus supercomputer. CHFS exhibits much better scalability and performance.

\section{CONCLUSION}

This paper proposed the design of a CHFS, an ad hoc temporal parallel file system for the persistent memory of compute nodes. It is based entirely on a highly scalable distributed key-value store with consistent hashing. It improves the scalability of parallel data access performance and metadata performance in terms of the number of compute nodes by not having dedicated metadata servers, sequential execution, and centralized data management. It was implemented to efficiently utilize multicore and manycore CPUs, high-performance networks, and remote direct memory access using the Mochi-Margo library.

The performance evaluation of persistent memory showed the advantage of pmemkv in the devdax mode. It displays $8.5 \mathrm{GiB} / \mathrm{s}$ for the put bandwidth in the devdax mode, while the POSIX write and pmemkv in the fsdax mode show $5.8 \mathrm{GiB} / \mathrm{s}$ and $4.2 \mathrm{GiB} / \mathrm{s}$, respectively.
Evaluating the performance of a 4-node persistent memory cluster, CHFS displays up to 9.9 times and 6.0 times better performance than DAOS and GekkoFS, respectively, on the IOR hard write benchmark. The performance is up to 6.0 times better than DAOS and 4.4 times better than GekkoFS on the MDtest hard write benchmark. The CHFS displays good scalability in terms of the number of compute nodes.

In the performance comparison with Gfarm/BB and BeeOND using 10 Cygnus supercomputer nodes, all file systems exhibited good performance on the IOR easy benchmark, while only CHFS displayed consistent good performance on the IOR hard benchmark. On the metadata benchmark, the CHFS exhibits 8.6 to 23.5 times better performance than Gfarm/BB and BeeOND except for the find benchmark; for this, it exhibits 3.1 to 3.2 times better performance than Gfarm/BB and BeeOND. These results demonstrate the low latency and high throughput of the metadata access of CHFS compared with those of Gfarm/BB and BeeOND.

Regarding scalability, CHFS displays better scalability and performance for both bandwidth and metadata compared with BeeOND and GekkoFS. When using 64 compute nodes, CHFS displays a 17.3 times better IO500 bandwidth score and a 15.8 times better IO500 metadata score compared to that of the one compute node case. Thus, CHFS is a good building block for HPC storage layers.

CHFS does not fully utilize the benefit of consistent hashing; however, it is useful to support redundancy for fault tolerance and checkpointing. Future work will focus on the redundancy support of CHFS.

\section{ACKNOWLEDGMENTS}

This work is partially supported by Multidisciplinary Cooperative Research Program in CCS, University of Tsukuba, a project (JPNP16007) commissioned by the New Energy and Industrial Technology Development Organization (NEDO), and Fujitsu Limited.

\section{REFERENCES}

[1] David Abramson, Chao Jin, Justin Luong, and Jake Carroll. 2020. A BeeGFS-Based Caching File System for Data-Intensive Parallel Computing. In Supercomputing Frontiers. SCFA 2020 (Lecture Notes in Computer Science, Vol. 12082). 3-22.

[2] J. Bent, G. Gibson, G. Grider, B. McClelland, P. Nowoczynski, J. Nunez, M. Polte, and M. Wingate. 2009. PLFS: a checkpoint filesystem for parallel applications. In Proceedings of the Conference on High Performance Computing Networking, Storage and Analysis. 1-12. https://doi.org/10.1145/1654059.1654081

[3] Peter Braam. 2019. The Lustre Storage Architecture. arXiv:1903.01955 [cs.OS]

[4] M. Scot Breitenfeld, Neil Fortner, Jordan Henderson, Jerome Soumagne, Mohamad Chaarawi, Johann Lombardi, and Quincey Koziol. 2017. DAOS for Extreme-scale Systems in Scientific Applications. arXiv:1712.00423 [cs.DC]

[5] André Brinkmann, Kathryn Mohror, Weikuan Yu, Philip Carns, Toni Cortes, Scott A. Klasky, Alberto Miranda, Franz-Josef Pfreundt, Robert B. Ross, and MarcAndré Vef. 2020. Ad Hoc File Systems for High-Performance Computing. Journal of Computer Science and Technology 35, 1 (2020), 4-26.

[6] Zhichao Cao, Siying Dong, Sagar Vemuri, and David H.C. Du. 2020. Characterizing, Modeling, and Benchmarking RocksDB Key-Value Workloads at Facebook. In 18th USENIX Conference on File and Storage Technologies (FAST 20). 209-223.

[7] DDN. 2021. Infinite Memory Engine. https://www.ddn.com/products/ime-flashnative-data-cache/

[8] Sanjay Ghemawat, Howard Gobioff, and Shun-Tak Leung. 2003. The Google File System. In Proceedings of 19th ACM Symposium on Operating Systems Principles. $20-43$.

[9] Shukai Han, Dejun Jiang, and Jin Xiong. 2020. SplitKV: Splitting IO Paths for Different Sized Key-Value Items with Advanced Storage Devices. In 12th USENIX Workshop on Hot Topics in Storage and File Systems (HotStorage 20).

[10] Kohei Hiraga, Osamu Tatebe, and Hideyuki Kawashima. 2020. Scalable Distributed Metadata Server Based on Nonblocking Transactions. Fournal of Universal Computer Science 26, 1 (2020), 89-106. 
[11] IO500. 2021. IO500 List. https://www.io500.org/

[12] David Karger, Eric Lehman, Tom Leighton, Matthew Levine, Daniel Lewin, and Rina Panigrahy. 1997. Consistent hashing and random trees: distributed caching protocols for relieving hot spots on the World Wide Web. In STOC '97: Proceedings of the twenty-ninth annual ACM symposium on Theory of computing. 654-663.

[13] Argonne National Labratory. 2019. ABT-IO: Argobots-aware bindings to POSIX I/O functions. https://github.com/mochi-hpc/mochi-abt-io/

[14] Siyang Li, Youyou Lu, Jiwu Shu, Yang Hu, and Tao Li. 2017. LocoFS: A LooselyCoupled Metadata Service for Distributed File Systems. In Proceedings of the International Conference for High Performance Computing, Networking, Storage and Analysis (SC'17). Article 4, 12 pages.

[15] J. Lofstead, I. Jimenez, C. Maltzahn, Q. Koziol, J. Bent, and E. Barton. 2016. DAOS and Friends: A Proposal for an Exascale Storage System. In SC '16: Proceedings of the International Conference for High Performance Computing, Networking, Storage and Analysis. 585-596. https://doi.org/10.1109/SC.2016.49

[16] Youyou Lu, Jiwu Shu, Youmin Chen, and Tao Li. 2017. Octopus: an RDMA-enabled Distributed Persistent Memory File System. In 2017 USENIX Annual Technical Conference (USENIX ATC 17). 773-785.

[17] Kelly Lyon. 2021. How Intel Optimized RocksDB Code for Persistent Memory with PMDK. https://software.intel.com/content/www/us/en/develop/articles/ how-intel-optimized-rocksdb-code-for-persistent-memory-with-pmdk.html.

[18] Adam Moody, Danielle Sikich, Ned Bass, Michael J. Brim, Cameron Stanavige, Hyogi Sim, Joseph Moore, Tony Hutter, Swen Boehm, Kathryn Mohror, Dmitry Ivanov, Teng Wang, Craig P. Steffen, and USDOE National Nuclear Security Administration. 2017. UnifyFS: A Distributed Burst Buffer File System - 0.1.0. https://doi.org/10.11578/dc.20200519.19

[19] Sarp Oral, Sudharshan S. Vazhkudai, Feiyi Wang, Christopher Zimmer, Christopher Brumgard, Jesse Hanley, George Markomanolis, Ross Miller, Dustin Leverman, Scott Atchley, and Veronica Vergara Larrea. 2019. End-to-End I/O Portfolio for the Summit Supercomputing Ecosystem. In Proceedings of the International Conference for High Performance Computing, Networking, Storage and Analysis (SC '19). Article 63, 14 pages.

[20] Swapnil Patil and Garth Gibson. 2011. Scale and Concurrency of GIGA+: File System Directories with Millions of Files. In Proceedings of the 9th USENIX Conerence on File and Storage Technologies. 177-190.

[21] Pmem.io. 2021. Persistent Memory Development Kit. https://pmem.io/pmdk/

[22] Pmem.io. 2021. Pmemkv. https://pmem.io/pmemkv/

[23] Yingjin Qian, Xi Li, Shuichi Ihara, Andreas Dilger, Carlos Thomaz, Shilong Wang Wen Cheng, Chunyan Li, Lingfang Zeng, Fang Wang, Dan Feng, Tim Süß, and André Brinkmann. 2019. LPCC: Hierarchical Persistent Client Caching for Lustre. In Proceedings of the International Conference for High Performance Computing, Networking, Storage and Analysis (SC '19). Article 88, 14 pages.

[24] Kai Ren, Qing Zheng, Swapnil Patil, and Garth Gibson. 2014. IndexFS: Scaling File System Metadata Performance with Stateless Caching and Bulk Insertion. In Proceedings of the IEEE/ACM International Conference for High Performance Computing, Networking, Storage and Analysis (SC14). 237-248.

[25] Robert B. Ross, George Amvrosiadis, Philip Carns, Charles D. Cranor, Matthieu Dorier, Kevin Harms, Greg Ganger, Garth Gibson, Samuel K. Gutierrez, Robert Latham, Bob Robey, Dana Robinson, Bradley Settlemyer, Galen Shipman, Shane Snyder, Jerome Soumagne, and Qing Zheng. 2020. Mochi: Composing Data Services for High-Performance Computing Environments. Fournal of Computer Science and Technology 35, 1 (2020), 121-144

[26] Takuto Sato, Osamu Tatebe, and Hiroyuki Kusaka. 2019. In-situ data analysis system for high resolution meteorological large eddy simulation model. In Proceedings of the 6th IEEE/ACM International Conference on Big Data Computing, Applications and Technologies. 155-158.

[27] Steve Scargall. 2020. Programming Persistent Memory. Apress.

[28] S. Seo, A. Amer, P. Balaji, C. Bordage, G. Bosilca, A. Brooks, P. Carns, A. Castelló, D. Genet, T. Herault, S. Iwasaki, P. Jindal, L. V. Kalé, S. Krishnamoorthy, J. Lifflander, H. Lu, E. Meneses, M. Snir, Y. Sun, K. Taura, and P. Beckman. 2018. Argobots: A Lightweight Low-Level Threading and Tasking Framework. IEEE Transactions on Parallel and Distributed Systems 29, 3 (2018), 512-526. https://doi.org/10.1109/ TPDS.2017.2766062

[29] Amazon Web Services. 2021. Amazon S3. https://aws.amazon.com/s3/

[30] J. Soumagne, D. Kimpe, J. Zounmevo, M. Chaarawi, Q. Koziol, A. Afsahi, and R. Ross. 2013. Mercury: Enabling remote procedure call for high-performance computing. In 2013 IEEE International Conference on Cluster Computing (CLUSTER). 1-8. https://doi.org/10.1109/CLUSTER.2013.6702617

[31] I. Stoica, R. Morris, D. Liben-Nowell, D. R. Karger, M. F. Kaashoek, F. Dabek, and H. Balakrishnan. 2003. Chord: a scalable peer-to-peer lookup protocol for Internet applications. IEEE/ACM Transactions on Networking 11, 1 (2003), 17-32. https://doi.org/10.1109/TNET.2002.808407

[32] Osamu Tatebe, Kohei Hiraga, and Noriyuki Soda. 2010. Gfarm Grid File System New Generation Computing 28, 3 (2010), 257-275.

[33] Osamu Tatebe, Shukuko Moriwake, and Yoshihiro Oyama. 2020. Gfarm/BB Gfarm File System for Node-Local Burst Buffer. Journal of Computer Science and Technology 35, 1 (2020), 61-71.
[34] ThinkParQ. 2018. BeeOND: BeeGFS On Demand. https://www.beegfs.io/wiki/ BeeOND

[35] Maarten van Steen and Andrew S. Tanenbaum. 2017. Distributed Systems (3rd ed.). distributed-systems.net.

[36] Marc-André Vef, Nafiseh Moti, Tim Süß, Markus Tacke, Tommaso Tocci, Ramon Nou, Alberto Miranda, Toni Cortes, and André Brinkmann. 2020. GekkoFS - A Temporary Burst Buffer File System for HPC Applications. Journal of Computer Science and Technology 35, 1 (2020), 72-91.

[37] T. Wang, K. Mohror, A. Moody, K. Sato, and W. Yu. 2016. An Ephemeral BurstBuffer File System for Scientific Applications. In SC '16: Proceedings of the International Conference for High Performance Computing, Networking, Storage and Analysis. 807-818. https://doi.org/10.1109/SC.2016.68

[38] Jian Yang, Joseph Izraelevitz, and Steven Swanson. 2019. Orion: A Distributed File System for Non-Volatile Main Memory and RDMA-Capable Networks. In 17th USENIX Conference on File and Storage Technologies (FAST 19). 221-234.

[39] Jian Yang, Juno Kim, Morteza Hoseinzadeh, Joseph Izraelevitz, and Steve Swanson. 2020. An Empirical Guide to the Behavior and Use of Scalable Persistent Memory. In 18th USENIX Conference on File and Storage Technologies (FAST 20). 169-182. 